\title{
Surgical Management of Traumatic Cerebrospinal Fluid Fistula in a Child with Recurrent Meningitis
}

\author{
Lília Ferraria ${ }^{1}$, Sílvia Alves ${ }^{2}$, Nélia Ferraria ${ }^{3}$, Mário Santos ${ }^{4}$, Helena Rosa $^{5}$, Luis Antunes ${ }^{6}$
}

\begin{abstract}
Aim: To report a case of a child with recurrent meningitis in which cerebrospinal fluid (CSF) leak diagnosis was established and treated by an extracranial approach.

Background: The diagnosis and management of pediatric posttraumatic CSF leak can be challenging. Untreated leaks can be manifested by intermittent rhinorrhea or recurrent meningitis, which can be the only form of presentation.

Case description: A 15-year-old girl had a past medical history of a fall from the second floor at the age of 8, which resulted in traumatic frontal sinus fracture. She had her first episode of pneumococcal meningitis at the age of 14 which led to the diagnosis of posttraumatic CSF fistula. External surgery was performed to repair the fistula, with no evidence of CSF rhinorrhea on postoperative examination. She was readmitted at the age of 15 years for a new episode of pneumococcal meningitis. The high-resolution computed tomography scan revealed an anterior and posterior wall defect of the right frontal sinus. An extracranial approach was performed with visualization of CSF trickling through the defect. Dural regeneration matrix and fascial graft were positioned on the defect, and fibrin glue was used. Frontal sinus obliteration was performed using hydroxyapatite with success.

Conclusion: The basic principles of pediatric CSF fistulas repair, such as an adequate exposure of the defect, do not differ from those of the adults. The successful repair of CSF leaks results in a preoperative correct identification of the fistula and bone defect site, an accurate knowledge of reconstruction techniques, and their judicious application in the procedure planning.

Clinical significance: This report documents a case of a child with recurrent meningitis as the only symptom of a CSF fistula as a consequence of a traumatic fall 7 years earlier. The CSF leaks' etiology, current diagnostic techniques, and surgical management are discussed.

Keywords: Cerebrospinal fluid leaks, Extracranial approach,, Pediatric trauma, Recurrent meningitis, Skull base defects.

Otorhinolaryngology Clinics: An International Journal (2019): 10.5005/jp-journals-10003-1332
\end{abstract}

\section{BACKGROUND}

A cerebrospinal fluid (CSF) fistula is a result of meningeal dural and arachnoid laceration with fistula formation, which may be spontaneous, secondary to a skull base fracture, related to surgical trauma, or associated with pathology of the skull base and/or secondary to a high-pressure system. ${ }^{1}$

The main cause of CSF fistula is secondary to a skull base fracture in $70-80 \%$ of the cases and most commonly originate from the cribiform plate, the fovea ethmoidalis, and the posterior wall of the frontal sinus or sphenoid sinus. CSF leaks appear in $6-13 \%$ of all cranial traumas involving the anterior skull base. ${ }^{1}$ While penetrating head trauma is an obvious cause of both transient and persistent CSF fistula, most traumatic CSF leaks occur as a result of blunt trauma due to the higher incidence of blunt head trauma overall. Posttraumatic CSF leaks in children are less common than in adults and occur in an incidence of $0.2-0.3 \%$ following childhood head trauma. This may be explained by the greater flexibility of skull bones that can lead to absorption of the traumatic shock. ${ }^{1}$

Clinically, posttraumatic CSF leaks can be manifested by intermittent rhinorrhea or recurrent meningitis, which can be the only form of presentation. ${ }^{2}$ Meningitis may develop acutely or even decades after the trauma. ${ }^{3,4}$ Overall, between $7 \%$ and $30 \%$ of all patients with posttraumatic CSF leakage will develop meningitisthis rate increases as the duration of CSF leakage increases. ${ }^{5}$

The diagnosis of a CSF leak after closed cranial trauma is based on the clinical evaluation, the immunofixation of beta-2-transferrin of the nasal drainage, the visual identification of a fistula and/or skull base defect (endoscopy), and its confirmation with a computed
1,2,4-6 Department of ENT, Hospital Garcia de Orta, Almada, Portugal ${ }^{3}$ Department of Pediatrics, Hospital Nossa Senhora do Rosário, Lisboa, Portugal

Corresponding Author: Lília Ferraria, Department of ENT, Hospital Garcia de Orta, Almada, Portugal, e-mail: liliaferraria@gmail.com

How to cite this article: Ferraria L, Alves S, Ferraria N, et al. Surgical Management of Traumatic Cerebrospinal Fluid Fistula in a Child with Recurrent Meningitis. Int J Otorhinolaryngol Clin 2019;11(1):15-18.

Source of support: Nil

Conflict of interest: None

tomography (CT) scan. The site of any defect should be defined using high-resolution coronal CT. This examination is the preferred test to detect defects at the cranial base. ${ }^{3}$

Most traumatic CSF leaks resolve spontaneously, the majority within the first 24-48 hours. Traumatic CSF leaks-as opposed to spontaneous ones - tend to stop spontaneously as a result of blood products and/or inflammatory adhesions at the site of the dural breach and associated skull fracture. Herniation of brain tissue into the traumatic defect may also play a role in leak cessation. However, persistent fistula does occur. ${ }^{5}$

Only a persistent CSF fistula becomes a significant management issue for the surgeon. The appropriate timing for surgical intervention in persistent posttraumatic CSF leakage is also controversial. What is clear is that many approaches can be successful, both intracranial and extracranial, depending on patient factors and the anatomy of the fistula. ${ }^{5}$ Recent studies indicate that 
the cumulative risk of developing meningitis within 10 years of a trauma is reduced from 8 to $7 \%$ when the surgery is successful. Consequently, there is general agreement that head-injured patients who present with enlarging pneumocephalus, persistent CSF leaks, or meningitis need urgent surgical repair. ${ }^{4}$

\section{Case Description}

A 15-year-old girl had a past medical history of a fall from the second floor at the age of 8 which resulted in a traumatic frontal sinus fracture. As there was no evidence of CSF rhinorrhea, she was not submitted to surgery at the time. Six years later, she was admitted to a pediatric hospital for an episode of pneumococcal meningitis and that leads to the diagnosis of CSF fistula due to the frontal sinus fracture. External surgery was performed in that hospital to repair the fistula. No evidence of CSF rhinorrhea on postoperative period was reported.

She was readmitted 1 year later, at the age of 15 years old, for a new episode of pneumococcal meningitis. She was transferred to our hospital, and the otorhinolaryngology department was consulted. Clinically, there was no evidence of CSF rhinorrhea, and through nasal endoscopy, we did not localize the site of the leak. Therefore, a high-resolution CT scan of the paranasal sinuses was requested. This examination revealed an anterior and posterior wall defect of the right frontal sinus (Figs 1 and 2). A fracture line in the posterior wall of frontal sinus created a communication with the intracranial space.

The child was treated by surgery under general anesthesia with endotracheal intubation. An extracranial approach via an eyebrow incision (Lynch-Howarth) was performed. The anterior wall of the frontal sinus was drilled with a cutting burr (Fig. 3). Meticulous attention was directed to the posterior table, where a CSF trickling was visualized (Fig. 4). The mucosa and inner periosteum were removed with a diamond drill. The exposed dura on the posterior wall of the frontal sinus was covered by a layer of dural regeneration matrix and a synthetic fascial graft-on-lay technique on the defect - with fibrin glue (Fig. 5). Separation of the frontal from the lower paranasal sinuses was achieved by placement of another synthetic fascial graft on the frontal recess for permanent occlusion.

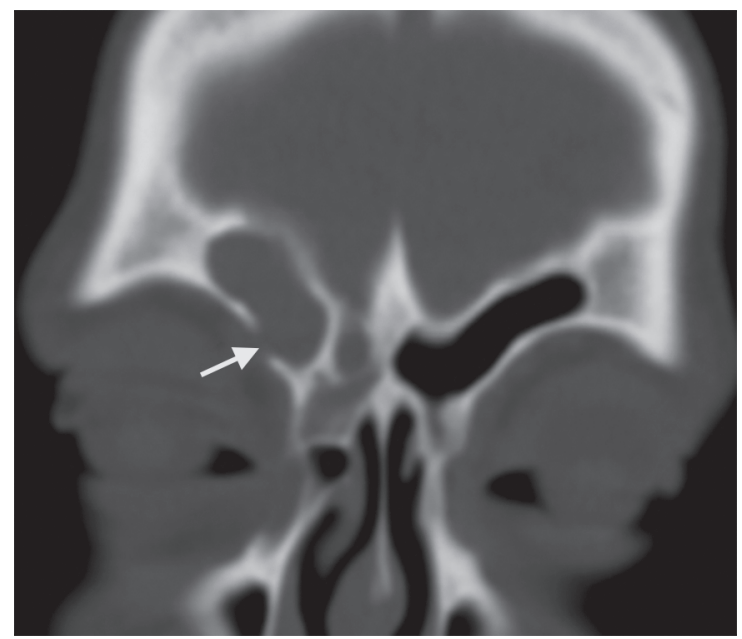

Fig. 1: Coronal computed tomography scan shows an anterior wall defect of the right frontal sinus (arrow)
Frontal sinus obliteration was performed using hydroxyapatite cement (Fig. 6).

Postoperatively, she had lumbar drain for 5 days, antibiotic therapy with ceftriaxone for 7 days, and bedrest with elevated head. She was discharged from the hospital 5 days postoperatively, and a regular follow-up was performed (Fig. 7).

Examination 22 months after the surgery revealed a well-healed incision on the right eyebrow and no evidence of CSF rhinorrhea. She reported no further episodes of meningitis.

\section{Discussion}

Diagnosis of CSF fistula is often based on clinical suspicion when there is a unilateral rhinorrhea with a history of previous surgery or head trauma. Rarely, recurrent meningitis may be the only indication that an abnormal communication with the nasal cavity exists. ${ }^{6}$

If there is CSF rhinorrhea, diagnosis of a CSF fistula is accomplished after nasal inspection and performance of protein electrophoresis-beta-2-transferrin test-in the nasal fluid, which demonstrates transferrin protein bands. ${ }^{7}$ This test is rapid and requires just few milliliters of liquid. It is the most sensitive laboratory test, when the rhinorrhea is active, for the presence of CSF in the nasal fluid with a sensitivity of about $98 \%{ }^{1,8}$ The limitation of this test is that rhinorrhea must be present. It is also sometimes difficult to collect the fluid sample, especially from pediatric patients. ${ }^{1}$ The dipstick test for glucose is not reliable, as both of these components can be seen in normal nasal and lacrimal secretions. ${ }^{8}$ In this case report, laboratory confirmation of the CSF fistula with Beta-2-transferrin was not possible to perform due to the absence of CSF rhinorrhea. The diagnosis of a CSF fistula was suspected based on clinical history of recurrent pneumoccocal meningitis.

The site of the transdural fistula must be located before attempting repair. Radiographic exams - simple skull X-rays-are ineffective. High-resolution CT in the axial and coronal planes is essential in identifying surgical anatomy and possible bony defects. Magnetic resonance imaging can be used as a noninvasive supplement to high-resolution CT in demonstrating herniated tissues. ${ }^{8}$ Contrast cisternography and intrathecal fluorescein dye

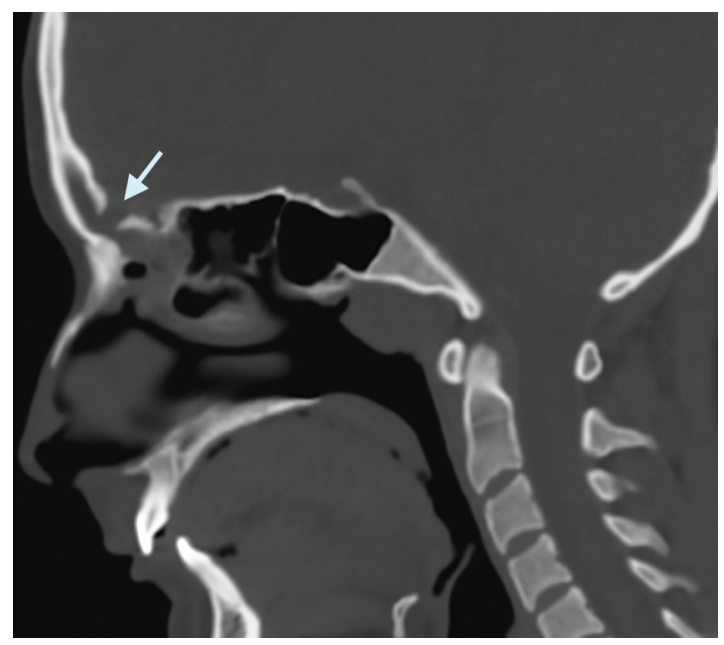

Fig. 2: Sagittal computed tomography scan shows a posterior wall defect of the right frontal sinus (arrow) that creates a communication with the intracranial space 


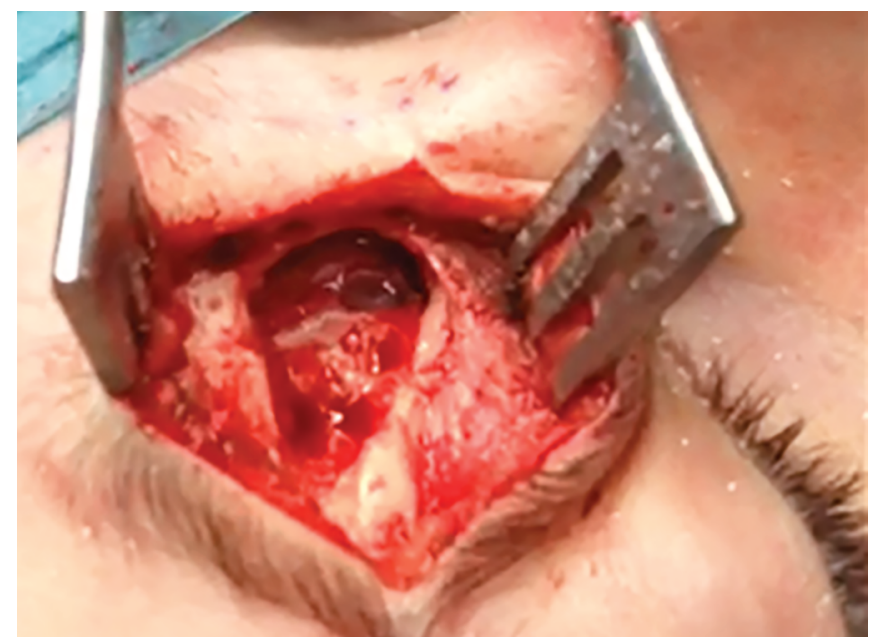

Fig. 3: An extracranial approach via an eyebrow incision (LynchHowarth). The anterior wall of the frontal sinus was drilled. Note the posterior table where a cerebrospinal fluid trickling is visualized

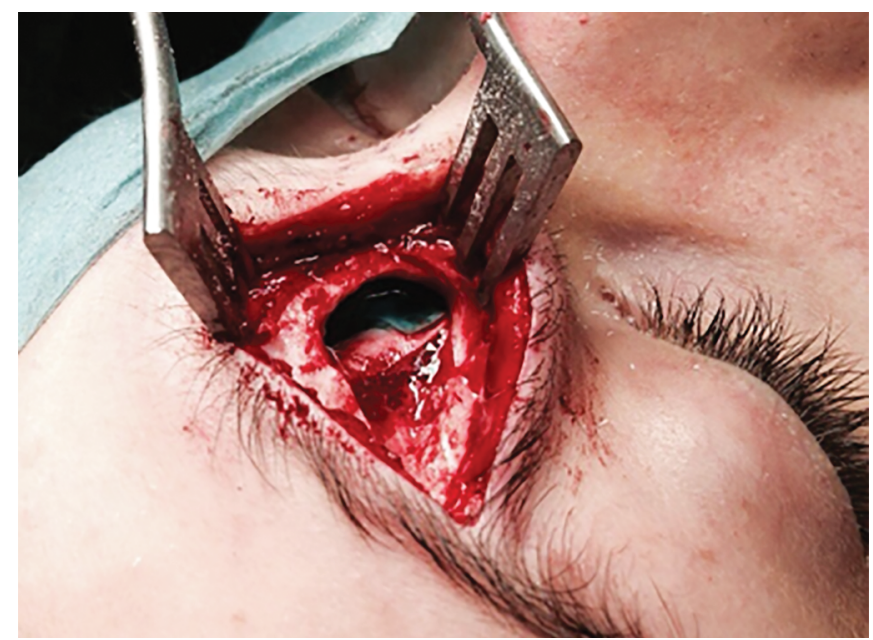

Fig. 5: Exposed dura on the posterior wall of the frontal sinus was covered by a layer of dural regeneration matrix and a synthetic fascial graft-on-lay technique on the defect

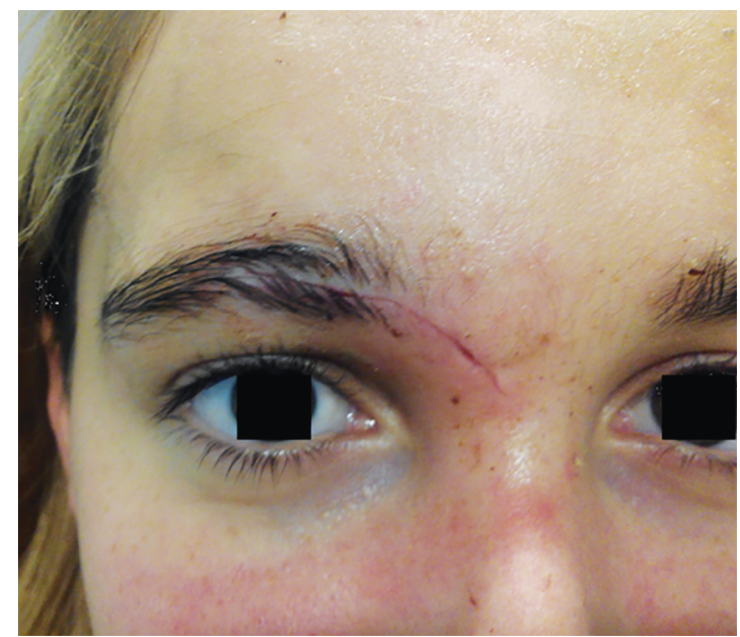

Fig. 7: Postoperative image: a well-healed incision on the right eyebrow

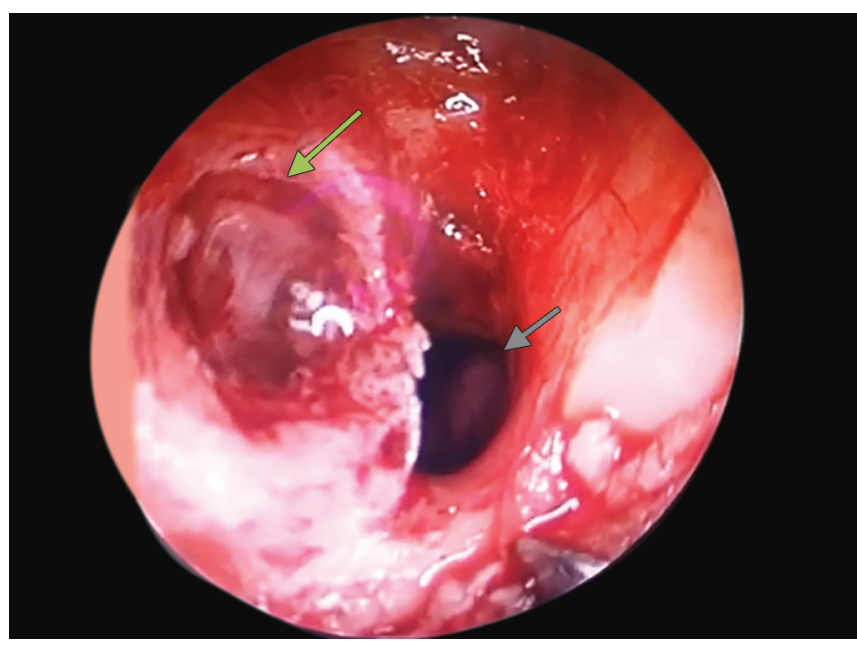

Fig. 4: Operative endoscopic view inside the frontal sinus-fistula site (green arrow) is identified with the cerebrospinal fluid coming out from the posterior wall of the frontal sinus; frontal recess (gray arrow)

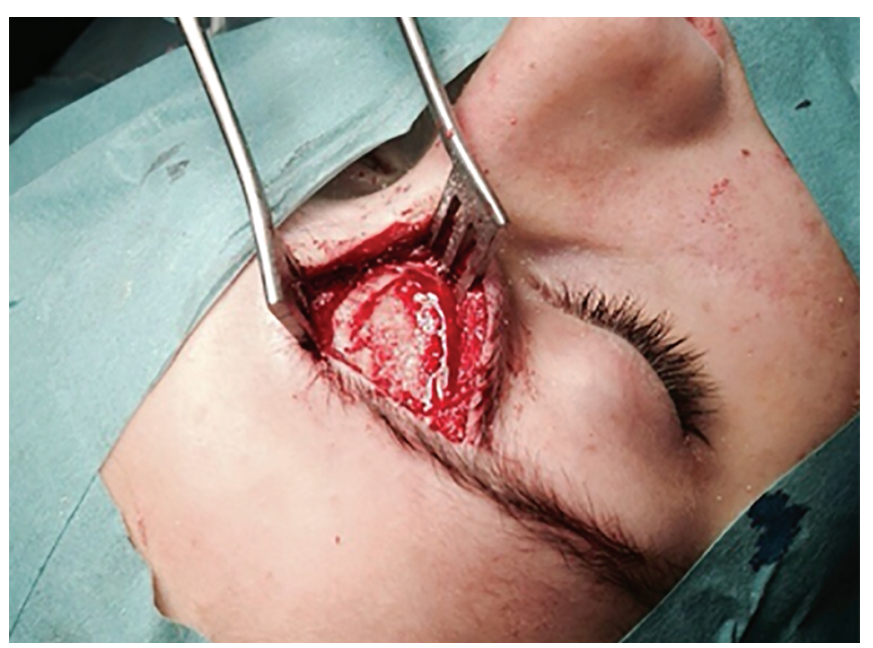

Fig. 6: Frontal sinus obliteration using hydroxyapatite cement

diluted in CSF may be confirmatory, but the CSF leak must be active at the time of evaluation. ${ }^{1}$ MRI cisternography is a method that does not require intrathecal injection of contrast and may become the method of choice for the evaluation of the CSF fistula. ${ }^{8}$ In this case report, the CSF leak was diagnosed through high-resolution CT scan of the paranasal sinus.

The majority of traumatic leaks resolve spontaneously within days of the injury. ${ }^{9}$ A surgical correction is required if persistent CSF rhinorrhea following acute trauma or recurrent meningitis are present. Repair is possible through intracranial, extracranial, or endoscopic approaches. Each technique offers its own indications, advantages, and disadvantages depending on the site of leak. ${ }^{10}$

As the child's CSF leak was localized in the posterior wall of the frontal sinus, the endoscopic repair was not possible due to reduced access and visibility. Only small defects of the area, which can be seen bulging anteriorly when the frontal recess has been opened, can be dealt with endoscopically. An extracranial approach was performed, minimizing the risk of intracranial complications. A Lynch-Howarth incision with removal of the anterior wall of the frontal sinus allows access to the defect. The site of the defect can 
then be defined and repaired with fascia and fat to hold the graft. A great number of techniques and materials have been used for CSF leak occlusion, including autologous material as abdominal fat, nasal septum mucosa, bone, fascia lata, and muscle grafts. The graft can be attached with fibrin glue, hemostatic sponges, or Vaseline gauzes. However, it appears that the location, size, techniques, and materials used do not interfere directly with the success of the procedure. A critical aspect of the procedure is an adequate resection of the mucosa around the bone defect in order to permit the complete graft attachment. ${ }^{8}$ In this case report, the graft consisted of dural regeneration matrix and a synthetic fascial graft fixed with fibrin glue. The graft can be positioned in an inlay form (between the dura and the skull base) or in an on-lay form (on the nasal site of the leak). Indication for performing the on-lay technique takes place when there is risk of damaging nerves and blood vessels during the dural detachment or during the intradural graft attachment. ${ }^{8}$ In this case, we performed the on-lay technique. Some studies report that the form of graft positioning is not a critical factor in predicting success of the procedure. ${ }^{11}$

Indications tp obliterate the frontal sinus with fat or hidroxyapatite cement are traumatic disruption of the frontal recess, advanced suppuration with epidural abscess or meningitis, loss of significant portions of the anterior table of the frontal sinus, and failure of endoscopic approaches to satisfactorily communicate the frontal sinus and the nasal cavity. ${ }^{12}$ In this case, the frontal sinus was obliterated with hydroxyapatite cement, which contoured the defect and filled the sinus.

Perioperative antibiotic was given, and an intradural lumbar drain was put in place. Some authors still recommend the routine use of a lumbar drain, although recent studies suggest it should not be routinely performed. ${ }^{8}$ Activity resulting in increased intracranial pressure, such as straining, bending, lifting, and nose blowing, are avoided for several weeks after surgery.

\section{ConcLusion}

Recurrent meningitis, especially pneumococcal, is usually caused by intracranial seeding of bacteria via a direct communication with an extracranial source. In an immunologically competent patient, recurrent meningitis should be considered a consequence of a transdural fistula until otherwise proven. The basic principles of pediatric CSF fistulas repair, such as an adequate exposure of the defect, do not differ from those of the adults. The successful repair of CSF leaks results in a preoperative correct identification of the fistula and bone defect site as well as an accurate knowledge of reconstruction techniques and their judicious application in the procedure planning. High-resolution $\mathrm{CT}$ in the axial and coronal planes is essential in defining bony anatomy and possible bony defects. The extracranial approach to anterior fossa CSF leaks can provide adequate exposure, virtually no central nervous system morbidity, and highly successful results.

\section{Clinical Significance}

This report documents a case of a child with recurrent meningitis as the only symptom of a CSF fistula as a consequence of a traumatic fall 7 years earlier. The CSF leaks etiology, current diagnostic techniques, and surgical management are discussed.

\section{References}

1. Ibrahim AA, Magdy EA, Eid M. Endoscopic endonasal multilayer repair of traumatic ethmoidal roof cerebrospinal fluid rhinorrhea in children. Int J Pediatr Otorhinolaryngol 2012;76(4):523-529. DOI: 10.1016/j.ijporl.2012.01.009.

2. Gupta AK, Gupta AK, Verma M. Cranionasal fistula in pediatric population: is endonasal endoscopic approach effective? Int J Pediatr Otorhinolaryngol 2007;71(10):1537-1541. DOI: 10.1016/ j.ijporl.2007.05.032.

3. Bernal-Sprekelsen M, Bleda-Vazquez C, Carrau RL. Ascending meningitis secondary to traumatic cerebrospinal fluid leaks. Am J Rhinol 2000;14(4):257-259. DOI: 10.2500/105065800779954473.

4. Yaldiz C, Ozdemir N, Yaman O, et al. Intracranial repair of posttraumatic cerebrospinal fluid rhinorrhea associated with recurrent meningitis. J Craniofac Surg 2015;26(1):170-173. DOI: 10.1097/SCS.0000000000001181.

5. Friedman JA, Ebersold MJ, Quast LM. Post-traumatic cerebrospinal fluid leakage. World J Surg 2001;25(8):1062-1066. DOI: 10.1007/ s00268-001-0059-7.

6. Marshall AH, Jones NS. Cerebrospinal fluid rhinorrhoea. In: Michael G, ed. Scott-Brown's Otorhinolaryngology, Head and Neck Surgery. Hodder Arnold; 2008. pp. 1636-1642.

7. Walz PC, Elmaraghy CA, Jatana KR. Endoscopic Skull Base Surgery in the Pediatric Patient, Endoscopy — Innovative Uses and Emerging Technologies. Associate Prof Somchai Amornyotin (Ed.), InTech; 2015. DOI: 10.5772/60555.Available from http://www.intechopen.com/ books/endoscopy-innovative-uses-and-emerging-technologies/ endoscopic-skull-base-surgery-in-the-pediatric-patient.

8. Landeiro JA, Flores MS, Lázaro BC, et al. Surgical management of cerebrospinal fluid rhinorrhea under endoscopic control. Arq Neuropsiquiatr 2004;62(3B):827-831. DOI: 10.1590/S0004282X2004000500016.

9. Ravindra VM, Neil JA, Shah LM, et al. Surgical management of traumatic frontal sinus fractures: case series from a single institution and literature review. Surg Neurol Int 2015;6:141. DOI: 10.4103/21527806.163449.

10. Das PT, Balasubramanian D. External frontal sinusotomy and endoscopic repair of cerebrospinal fluid fistula in the posterior wall: preliminary report of a new technique. J Laryngol Otol 2011;125(8):802-806. DOI: 10.1017/S0022215111001150.

11. Zweig JL, Carrau RL, Celin SE, et al. Endoscopic repair of cerebrospinal fluid leaks to the sinonasal tract: predictors of success. Otolaryngol Head Neck Surg 2000;123(3):195-201. DOI: 10.1067/mhn.2000. 107452.

12. Petruzzelli GJ, Stankiewicz JA. Frontal sinus obliteration with hydroxyapatite cement. Laryngoscope 2002;112(1):32-36. DOI: 10.1097/00005537-200201000-00006. 